\title{
Periodismo especializado en medio ambiente: el caso Doñana como paradigma de manipulación informativa
}

\author{
Dr. Carlos Elías \\ Profesor de Periodismo Especializado de \\ la Universidad Carlos III de Madrid
}

\begin{abstract}
RESUMEN
Este artículo es parte de una tesis doctoral titulada "Flujos de información entre científicos y prensa" en la que, entre otros asuntos, se aborda la manipulación política de la información científica. En esta tesis se utilizó la metodología de investigación participativa, que implicó, entre otras circunstancias, que quien suscribe este artículo estuviera seis meses en el gabinete de prensa del CSIC como becario para investigar a fondo cómo se gestiona la información desde un gabinete de prensa gubernamental. En el artículo se demuestra la gran influencia de los gabinetes de comunicación en la prensa española. También se analiza cómo se manipula el mensaje y cómo se seleccionan las fuentes para someter los criterios científicos a los políticos. Para demostrar esta hipótesis se han estudiado todas las informaciones que aparecieron sobre el desastre ecológico de Doñana en la prensa de tirada nacional desde abril hasta julio de 1998. Se han relacionado las diferentes actuaciones del gabinete con la teoría general de la gestión de crisis informativas.
\end{abstract}

\section{ABSTRACT}

This research are included in a doctoral thesis about information flows between scientists and press. We use participative research methods. For that, the author got a fellowship of CSIC, the biggest spanish research center, and worked as a member of its press office during a six months periods. This article shows how scientific press offices have a very big influence in spanish press. Also the research proves how messages is manipulated and how the scientific sources are selected in order to indentify scientific and politics criteria. For demostrate how this manipulation is done, I analyse press information about Doñana ecological catastrophe published in 1998 in spanish national newspapers from april to july. Also these dates proves the relationship with general theories about informative crisis.

Palabras clave: Doñana/Periodismo especializado/Crisis informativas/Gabinetes de prensa/Periodismo medioambiental.

Key Words: Doñana/Specialized jornalism/Informative crises/Press offices/Environmental journalism. 


\section{El estallido de la crisis: crónica de los acontecimientos}

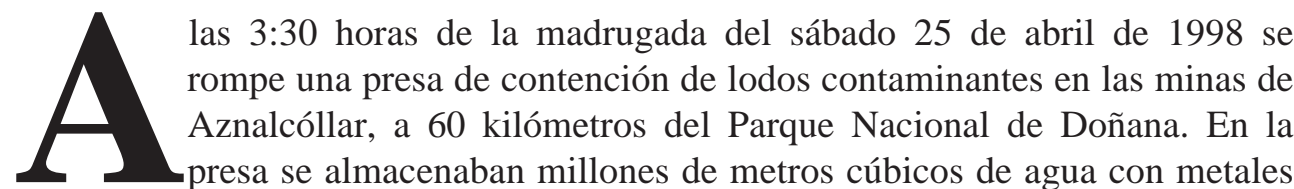
pesados. Ese mismo día la ministra de Medio Ambiente, Isabel Tocino, visita la zona afectada. El domingo 26 de abril se improvisan unos diques para impedir que el agua contaminada se extienda por el parque nacional, pero aún así quedan arrasadas 5.000 hectáreas de cultivos en el entorno. Ese día el acontecimiento es primera página de todos los periódicos españoles ${ }^{1}$. Las televisiones, las emisoras de radio y las agencias de noticias habían dado la noticia desde el día anterior. La crisis había estallado y los medios de comunicación estaban ávidos de información. El 27 de abril ya aparecieron declaraciones en las que el Ministerio de Medio Ambiente, del PP, y la Junta de Andalucía, del PSOE, eludían sus culpas respectivas y se cruzaban acusaciones mutuas sobre la responsabilidad del vertido.

A esas alturas de la crisis ya se conocía la responsabilidad de cada actor de esta tragedia. Los inclinómetros que poseía la presa y cuya misión era calibrar si los muros de la misma cedían ante la presión del agua tóxica embalsada, propiedad de la empresa sueca Boliden Apirsa, no funcionaban o estaban sucios y no medían adecuadamente las inclinaciones de los muros. Aun así se solicitó permiso para desembalsar 1 millón de metros cúbicos de agua al Guadiamar, afluente del Guadalquivir, pero la Confederación Hidrográfica del Guadalquivir lo prohibió, pues no podía permitir ese vertido sin que antes hubiese sido depurado.

La Confederación Hidrográfica es la encargada de vigilar los vertidos al río y pertenece al Ministerio de Medio Ambiente. La Consejería de Industria de la Junta de Andalucía, del PSOE, era la encargada de vigilar la seguridad de la balsa, un cometido que diseñó y ejecutó una empresa denominada Geocisa. Esta empresa debía controlar, entre otros aspectos, el buen funcionamiento de los inclinómetros. La Consejería de Medio Ambiente, también del PSOE, debía controlar los residuos tóxicos pero no los mineros.

El 20 de abril, cinco días antes del suceso, Geocisa remitió a la Consejería de Industria un informe -concluido el 31 de marzo y publicado en El País (17 de mayo)- en el que se alertaba del deficiente estado de los cuatro sensores de movimientos del muro. Días después, la Consejería de Industria, según su director general, Francisco Mencia, solicitó a esta empresa que lo reparase. Geocisa no informó sobre si cumplió la reparación. Ningún inspector lo verificó. Boliden

1 Aparece en primera página de todos los diarios españoles, a pesar de que competía con otra noticia importante: el 25 de abril, José Borrell ganaba las primarias del PSOE y se convertía en el candidato socialista a la presidencia del gobierno, en lugar de Joaquín Almunia. 
Apirsa no interrumpió su producción. Ninguna autoridad lo exigió. Continuó el llenado de la balsa y subió el volumen del agua y la presión de los lodos tóxicos. El día 25 de abril ya no hicieron falta los inclinómetros para saber que los muros se movían.

El 28 de abril el director de Estación Biológica de Doñana, Miguel Ferrer, señala a la prensa que el acuífero está afectado. La Estación Biológica ${ }^{2}$ pertenece al CSIC y su director, un cargo de confianza del presidente. Inmediatamente fue llamado a Madrid y se le prohibió realizar más declaraciones sin consultar antes con el CSIC.

El Ministerio de Medio Ambiente estaba apareciendo en la prensa como culpable cuando, en realidad, los responsables de que las inspecciones no se hubiesen llevado a cabo eran los consejeros de Industria y Medio Ambiente de la Junta de Andalucía. El gabinete de prensa del presidente de la Junta, Manuel Chaves, dirigió desde el primer momento una ofensiva muy fuerte contra Isabel Tocino.

La estrategia del Ministerio de Medio Ambiente estuvo, en un principio, muy mal diseñada para combatir una crisis. Fue a la defensiva y no tomaba la iniciativa. Esto fue un error según los últimos estudios sobre gestión de crisis informativas. Así, el investigador en comunicación científica Pierre-Marie Fayard ${ }^{3}$ señala:

"La energía liberada por una crisis alimenta la ruptura del consenso previo entre las partes de un todo hasta entonces en equilibrio 'a falta de algo mejor'. Adoptar una postura defensiva o a la espera de acontecimientos provoca el abandono de la escena de acción y de la iniciativa a merced de otros principios de existencia, mientras otros actores, no necesariamente bien intencionados, están a la espera de que la crisis empiece a hacer efecto".

En parte, la culpa de esta falta de estrategia la tenía el hecho de que la ministra había cambiado frecuentemente a su jefe de prensa durante su mandato. La estrategia de comunicación de la Junta de Andalucía apelaba a los sentimientos. "La ministra sólo viene a hacerse la foto", declaraba Manuel Chaves dos días después del desastre y tras la visita de Isabel Tocino.

2 La Estación Biológica se creó en 1974 pero antes, en 1963, el CSIC fundó la Reserva Biológica de Doñana, germen de la actual estación.

3 Pierre-Marie Fayard dictó una conferencia sobre la estrategia de comunicación en situación de crisis en el curso sobre Comunicación de Crisis. IDEC, Universidad Pompeu Fabra. Abril, 1997. Barcelona. Esta conferencia fue recogida en el no 10 de la revista Quark correspondiente a enero-marzo de 1998 (pp. 70 80). 
Mientras, la estrategia de comunicación del ministerio cometía un error muy frecuente: apelar a la razón. Las declaraciones sólo giraban en el sentido de que el ministerio no tenía competencias y la Junta sí. Pronto se comprobó que esa política no beneficiaba al ministerio, porque los responsables de comunicación de la Junta contraatacaban con similares razonamientos y era obvio que ni los periodistas ni la población iba a perder el tiempo en averiguar a quién correspondían las competencias.

Incluso llegaron a criticar a la ministra por decir que el parque nacional de Doñana se había salvado ${ }^{4}$. La afirmación era cierta. El parque nacional, responsabilidad del Ministerio de Medio Ambiente, no había sido afectado por la contaminación, al menos en su superficie, porque luego se comprobó que los acuíferos que lo surten sí estaban contaminados, pero esta verificación se realizó el 29 de abril, dos días después de las declaraciones de la ministra. Lo que sí estaba afectado era el parque natural de Doñana, que pertenecía a la Junta. Sin embargo, pocos periodistas y menos lectores estaban al tanto de la diferencia entre parque nacional y parque natural, entre parque y preparque. Estaba claro que la estrategia de apelar a la razón había que aplicarla de otra forma.

La estrategia de iniciativa y de declaraciones sentimentales que tuvo la Junta de Andalucía en un primer momento tuvo también sus fallos conceptuales como cuando el consejero de Medio Ambiente arremetió contra la Confederación Hidrográfica por bombardear agua contaminada al río Guadiamar. Pero no matizó que habían sido los propios técnicos de la Junta, es decir, sus subordinados, los que habían sugerido tal decisión, para evitar una mayor avalancha de lodos. Sin embargo, lo importante era tomar la iniciativa y "echar la culpa al ministerio".

Un rosario de políticos visitó Doñana para apoyar a uno u otro bando. La estrategia ahora era estar en los medios de comunicación para justificar y argumentar las posturas de cada partido político y recriminar las del adversario. Por el PP, visitaron el parque, entre otros, el presidente de gobierno, José María Aznar; el ministro de Industria, Josep Piqué; la titular de Agricultura, Loyola de Palacio, y el de Trabajo, Javier Arenas, quien también era el responsable en aquella época del PP en Andalucía. Por el PSOE acudieron desde los miembros de la Junta hasta el candidato a la presidencia de gobierno en aquella época, Josep Borrell.

\section{Reconducción de la crisis hacia el plano científico}

Estaba claro que en el plano político no se solucionaba nada porque éstos no se ponían de acuerdo y sus declaraciones estaban mediatizadas por el partido

4 En concreto en las declaraciones, realizadas en Madrid el día 26 de abril, la ministra de Medio Ambiente señaló: "El 25 fue un día muy triste, pero el corazón de Doñana se ha salvado". Esta opinión fue recogida por toda la prensa nacional el 27 de abril. 
al que pertenecían. En el plano científico el asunto también amenazaba con salirse de los cauces previstos. En este caso, tanto el gobierno como la Junta querían que los científicos aseguraran que los daños no habían sido muy graves, de forma que sus mutuas responsabilidades quedaran rebajadas. Sin embargo, la opinión de los científicos independientes era muy crítica. De ahí surgió la idea de encauzar todas las opiniones científicas a través de un organismo científico susceptible de ser manipulable políticamente como el CSIC.

Al revisar los periódicos de esos días se demuestra cómo en un primer momento todos los científicos españoles que sabían algo sobre el tema pudieron opinar. Sólo cuando el gabinete de prensa del CSIC tomó las riendas del asunto, se observa que el CSIC cobró el absoluto protagonismo científico, al tiempo que otros expertos dejaron de aparecer.

\section{Diferencias entre los tres diarios nacionales en el tratamiento informativo de la crisis}

Si estudiamos las repercusiones de la noticia en los tres diarios de tirada nacional -El País, Abc y El Mundo- observamos bastantes diferencias en cuanto al tratamiento de la información.

El diario El Mundo fue, sin duda, el que mejor trató la información, no sólo desde el punto de vista de su cobertura informativa -número de páginas-, sino de su independencia de criterio así como por la inclusión desde un primer momento de las opiniones científicas, algo que no hicieron los otros dos.

El domingo día 26 de abril El Mundo titulaba a 4 columnas en primera página "Desastre ecológico a las puertas de Doñana", al tiempo que movilizaba desde el mismo día 25 a su redactor especializado en medio ambiente -Gustavo Catalán Deus- quien apoyado por otro redactor y la corresponsal del diario en Sevilla cubrieron el accidente ecológico durante las primeras semanas.

El día 27 El Mundo ya incluyó un artículo del profesor de Economía de la Universidad de Zaragoza Pedro Arrojo, quien además era miembro por España del Comité Hombre-Biosfera.

Las primeras declaraciones de un científico en la prensa española sobre este asunto se produjeron en El Mundo el 29 de abril. Correspondían al presidente de la Real Academia de Ciencias, Angel Martín Municio, y aparecieron en la portada del citado diario.

En ellas, Martín Municio aseguraba, entre otras afirmaciones: "La repercusión de este vertido será para la eternidad en miles de hectáreas" y añadía: "La sociedad española no se está dando cuenta de la brutal y terrible trascendencia de esta contaminación ambiental".

El mismo día 29 también aparecen en El Mundo las opiniones de Miguel Ferrer, en su calidad de director de la Estación Biológica de Doñana, no de científico; y de los científicos Narcís Prats, catedrático de Ecología de la Univer- 
sidad de Barcelona, y de Miguel Delibes de Castro $^{5}$, director de la Estación Biológica desde 1988 hasta 1995 y estudioso del ecosistema de Doñana durante 25 años, el cual vaticinaba: "El agua y el lodo formarán una bomba permanente de tierra apestada que se irá filtrando poco a poco".

Estas declaraciones eran ampliadas por la opinión del científico del CSIC Román Román, del Instituto de Ciencias Medioambientales, quien explicaba a $E l$ Mundo (29 de abril): "La concentración de contaminantes en el acuífero es baja en estos momentos porque éste está cargado de agua, pero aumentará en el verano, cuando el volumen de agua comience a descender".

El diario El Mundo fue también el más crítico tanto con el PP como con el PSOE en este asunto. Pese a lo que pueda creerse respecto a la afinidad de este periódico con el PP, las críticas más duras contra Isabel Tocino aparecieron en este diario. Así, en su editorial del 30 de abril El Mundo publicaba:

“Isabel Tocino minimizaba el desastre con alegre precipitación 'Doñana está salvado'. En lugar de permanecer a pie de obra (...) ha estado fuera de España dos días para firmar el protocolo de Kioto, un documento que podría haber signado en su nombre el secretario de Estado. (...) Su viaje transoceánico suena a espantada y a dejación de responsabilidades". (El Mundo, 304-1998: 3)

En el mismo editorial además de responsabilizar a la Junta de Andalucía de sus dejaciones, también se recuerda que "José Borrell estaba al frente de Obras Públicas, ministerio del que dependía Medio Ambiente, cuando se produjeron las primeras denuncias en 1995".

El diario El País titula en la primera página del domingo 26 de abril: "La rotura de una presa con agua ácida causa un desastre ecológico en Doñana" pero no desplazó a su redactor especializado en medio ambiente -Inmaculada Mardones-. La información del domingo se elaboró con agencias y con la corresponsal del diario en Sevilla.

El día 27 de abril El País envía a un redactor desde Madrid, pero no al especializado en medio ambiente, sino a uno de los de la sección de ciencia Javier Sampedro-. Es interesante destacar asimismo que hasta el 30 de abril, en que aparece la reunión de los científicos del CSIC del día 29, El País no incorpora a su información la opinión de ningún científico. Sólo aparecen declaraciones de miembros del gobierno -desde ministros a directores generales-; de la Junta -

5 Miguel Delibes de Castro comenzó a trabajar en su tesis doctoral sobre la ecología del lince ibérico de Doñana en 1974, tras colaborar con Félix Rodríguez de la Fuente en la enciclopedia Salvat de la vida salvaje española. Su director de tesis fue Javier Castroviejo. En la actualidad investiga sobre el lince y la nutria de Doñana. 
desde consejeros hasta técnicos-; de alcaldes de las zonas afectadas y de organizaciones ecologistas. Sin embargo, no aparecen opiniones y, sobre todo, evaluaciones de ningún científico ${ }^{6}$.

Esta estrategia de El País, utilizada en los primeros días, de no incorporar opiniones independientes a la de los poderes establecidos creo que se sustenta en el hecho de que posiblemente debió sufrir fuertes presiones de los dirigentes del socialismo andaluz que salían muy perjudicados en declaraciones de científicos que no sólo los responsabilizaban, sino que en vez de minimizar las consecuencias de la catástrofe -tal y como pretendían la Junta y el gobierno- las maximizaban.

Sin embargo, a medida que transcurrieron los días El País fue introduciendo la opinión de otros científicos. Destaca la de Miguel Delibes (9 de mayo) y la no inclusión de las del presidente de la Real Academia de Ciencias.

No obstante, el artículo de Miguel Delibes es bastante crítico. En él se señala:

"La balsa de lodos tóxicos y aguas ácidas de las minas de Aznalcóllar se rompió y los efectos de la negra riada consiguiente empiezan, sólo empiezan, a ser conocidos: desolación, muerte, impotencia, miedo a lo que ocurra en el futuro, a cuánto tiempo pueda prolongarse la desgracia”. (El País, 95-1998: 12)

Tras este análisis, criticaba a ministra Isabel Tocino señalando que no pueden cavarse trincheras y distribuir fuerzas a las puertas del parque nacional, por si acaso, mientras se destruyen el parque natural y su entorno. Añadía: "Mucho menos, por supuesto, se puede presumir luego de que 'lo mío se ha salvado'. De poco vale que el corazón de Doñana quede indemne si se queman sus brazos y sus piernas".

Sin embargo, Delibes no critica en la misma medida la dejación de responsabilidades de la Junta en cuanto a la inspección de los inclinómetros. Sólo apunta: "La disputa entre los responsables de los gobiernos central y autonómico recuerda a la de dos niños que, jugando, rompieran el jarrón chino de la estantería familiar".

El País le dedica al accidente dos páginas los días 26 y 27; cuatro el 28 y tres y un editorial el 29. En el editorial titulado "Escurrir el bulto" se critica el mutuo cruce de acusaciones entre la Junta y el Ministerio de Medio Ambiente, pero la crudeza de la crítica es inferior a la de El Mundo.

6 El día 29 de abril El País publica una entrevista con el director de la estación Biológica de Doñana, Miguel Ferrer, en la que asegura que el acuífero 27 que surte de agua al parque nacional de Doñana está contaminado. Estas declaraciones son publicadas por el resto de los medios de comunicación y si bien tiene un contenido científico, Ferrer las hace en función de su cargo político como director de la estación biológica. 
$A b c$ publicó un editorial el mismo día 26 de abril en el que instaba "a buscar soluciones en lugar de eludir responsabilidades". Destaca que, al igual que El País, tampoco envía a sus redactores especializados en medio ambiente a Sevilla, sino que la información se elabora desde allî́. Al igual que en El País, en $A b c$ no aparece la opinión de los científicos hasta el día 30 de abril, es decir la referida a la reunión de los "expertos" del CSIC. Las fuentes que aparecen en las noticias proceden del gobierno central, de la Junta de Andalucía, de técnicos y de ecologistas. $A b c$, acorde con su línea ideológica, prima en los titulares las declaraciones de la ministra de Medio Ambiente. Titulares como "Tocino: el corazón de Doñana se ha salvado" (27 de abril) o "Isabel Tocino apunta directamente a la Junta de Andalucía como responsable de los vertidos" (28 de abril) ilustran esta hipótesis.

Durante los primeros días esta información no aparece en la portada de $A b c$, aunque es necesario matizar que las portadas de este diario no siguen los criterios habituales de otros periódicos debido a la particularidad de incluir una foto en color de grandes dimensiones en la misma.

Tras este análisis sobre cómo se desarrolló la noticia en los primeros días puede concluirse que en los momentos iniciales los periodistas no sabían a qué científico acudir o no consideraron necesario incluirlos en la información, un hecho habitual en la información medioambiental española, como ya se ha señalado en esta tesis.

Uno de los primeros en ser contactado fue el presidente de la Real Academia de Ciencias, Ángel Martín Municio, quien dio su opinión en varias emisoras de radio y televisión. Incluso en un reportaje publicado en El Mundo (1 de mayo) titulado "Las claves del mayor desastre medioambiental", la opinión científica proviene sólo de Martín Municio. A la pregunta del periodista sobre “¿Hasta cuándo permanecerá la contaminación?", el presidente de la Real Academia de Ciencias le contesta: "Eternamente". Y añade:

"Esto quiere decir que la recarga de contaminantes en el acuífero de Doñana, que tiene aguas fósiles de cientos de años, darán trazas de metales pesados dentro de varios siglos. Cualquier análisis profundo de las tierras afectadas también detectará durante décadas el vertido". (El Mundo, 1-51998: 25)

Otro de los científicos al que también se le consultó sobre el desastre fue a Miguel Delibes de Castro quien defendió a los ecologistas en varias emisoras de radio y televisión. Sus declaraciones fueron recogidas en El País (4 de mayo) en

7 Debe matizarse que en el caso de $A b c$, la redacción de Sevilla no es una simple corresponsalía, sino que tiene casi tantos periodistas como la de Madrid. 
un artículo recopilatorio. Bajo el título "Delibes: los ecologistas tenían razón”, el que fuera director de la Estación Biológica de Doñana afirmaba: "En su momento nos convencieron (los técnicos del gobierno y la Junta) de que esto no podía ocurrir nunca, y nosotros nos dejamos convencer". Para subrayar a continuación: "Los únicos en levantar la voz fueron los ecologistas".

Delibes se refería a los informes que la Confederación Ecológica Pacifista de Andalucía (CEPA) remitió en enero de 1996 a la Unión Europea y en los que se apuntaba también la existencia de filtraciones. Tanto el gobierno como la Unión Europea negaron posibles impactos ecológicos. Tras esta denuncia, un ex técnico de la empresa Boliden Apirsa denunció también la existencia de filtraciones de los residuos que almacenaba la presa minera al río Agrio, afluente del Guadiamar, que a su vez surte de agua a Doñana. El juzgado número dos de Sanlúcar la Mayor (Sevilla) archivó el caso a principios de 1998 al no apreciar "hechos constitutivos de delitos", a pesar de que en el escrito constaba que "las filtraciones de residuos almacenados en la balsa eran debido a la mala ejecución del recrecimiento de las paredes de la presa minera".

El ex director de la Estación Biológica de Doñana Miguel Delibes criticó, en la entrevista mencionada de El País, la decisión judicial y argumentaba que la misma debe servir para crear un órgano judicial específico para delitos contra el medio ambiente".

Para rematar la entrevista, Delibes acusaba a la Junta y al gobierno central de no haber actuado y desmantelaba el principal argumento científico del gobierno para eludir responsabilidades al asegurar que es "absurda" la dicotomía entre parque nacional y parque natural, ya que sus mecanismos ecológicos están muy intrincados. "Lo que está en juego -añadía- es la comarca de Doñana" y finalizaba diciendo: "Los contaminantes vertidos no matan inmediatamente, pero van a lleva a una reducción de la calidad y diversidad de fauna en los próximos 20 años".

Con este análisis se puede concluir que en los primeros momentos, los periodistas sí iban bien encaminados en cuanto a buscar a fuentes científicas acreditadas. Sólo cuando entró en acción un gabinete de prensa tan potente como el del CSIC quedaron apabullados y aceptaron lo que de él provenía. De hecho, si se analizan las informaciones publicadas en la prensa a partir del 30 de abril se observa que la práctica totalidad de la opinión científica proviene de los denominados "expertos" nombrados por el CSIC.

\section{La oportunidad del CSIC de intervenir en la crisis}

Aunque no se actuó desde el principio, desde los primeros momentos en el gabinete de prensa del CSIC se vislumbró la posibilidad de canalizar toda la información científica a través de él. Tomó cuerpo la idea de formar un grupo de expertos -en realidad, no en Doñana, daba igual el tema- de los cuales el portavoz y la cabeza visible fuera siempre el presidente del CSIC. Periodísticamente era 
irrelevante que los expertos lo fueran en Doñana o en el genoma humano. Los periodistas no iban a reparar en eso. Aunque científicamente resultaba un disparate que investigadores de áreas no relacionadas con Doñana tomaran decisiones, políticamente resultaba una gran ventaja, pues los expertos seleccionados eran "amigos" del presidente, César Nombela ${ }^{8}$ al cual desde el Ministerio de Medio Ambiente se le había sugerido que defendiera con más ahínco la postura gubernamental y que apareciera más en la prensa, pues el único que estaba saliendo en los medios -en los primeros días de la crisis- era Martín Municio.

El comité de expertos se reunió por primera vez en la tarde del 29 de abril. Formado por 17 personas $^{9}$, todas ellas doctores, en él estaban desde César Nombela, experto en genética microbiana, hasta el director de la Estación Biológica de Doñana, Miguel Ferrer, cuyo cargo es propuesto por el presidente del CSIC.

Muchos investigadores que, incluso, habían dirigido proyectos de investigación financiados por la Comisión Interministerial de Ciencia y Tecnología relacionadas con Doñana no estaban en el comité. Destacaba la ausencia de dos investigadores importantes del CSIC relacionados con Doñana: Miguel Delibes y Javier Castroviejo $^{10}$, primer director de la Estación Biológica de Doñana, cargo que ostentó durante 14 años y considerado la máxima autoridad mundial en el ecosistema del parque.

8 César Nombela fue sustituido en su cargo de presidente del CSIC por Rolf Tarrach en octubre de 2000. Nombela recibió el 23 de abril de 2001, justo unas horas antes del tercer aniversario del Desastre Ecológico de Doñana, la Gran Cruz del Mérito Civil, otorgada a propuesta del Ministerio de Asuntos Exteriores. El acto estuvo presidido por la ministra de Ciencia y Tecnología, Anna Birulés.

9 Los miembros del comité de expertos eran los siguientes: César Nombela, presidente del CSIC; Miguel García Guerrero, vicepresidente de Organización y Relaciones Institucionales del CSIC; Teresa Mendizábal, vocal asesora de Presidencia del CSIC; Miguel Ferrer, director de la Estación Biológica de Doñana (CSIC); Enrique Macpherson, coordinador científico del área de Recursos Naturales del CSIC; José Juan Chans, investigador de la Estación Biológica de Sevilla; Víctor de Lorenzo, del Centro Nacional de Biotecnología (CSIC); Juan Luis Ramos, de la Estación Experimental del Zaidín (Granada) (CSIC); Juan Cornejo, Instituto de Recursos Naturales y Agrobiología de Sevilla (CSIC); María Jesús Sánchez, del Instituto de Recursos Naturales y Agrobiología de Salamanca (CSIC); Francesc Gallart, del Instituto de Ciencias de la Tierra Jaume Almera de Barcelona (CSIC); Luis Hernández Saint-Aubin, del Instituto de Química Orgánica General de Madrid (CSIC); Josep María Bayona, del Centro de Investigación y Desarrollo de Barcelona (CSIC); Rosa Montoro, del Instituto de Agroquímica y Tecnología de Alimentos de Valencia (CSIC); Francisco Cabrera, Instituto de Recursos Naturales y Agrobiología de Sevilla (CSIC) y Julio Camargo de la Universidad de Alcalá de Henares (Madrid). Asistió como invitado Javier Cobos, director del Parque Natural de Doñana (Junta de Andalucía) y fue invitado pero no pudo asistir a la reunión Alberto Ruiz de Larramendi, director-conservador del Parque Nacional de Doñana (Ministerio de Medio Ambiente).

10 Castroviejo escribió uno de los escasos artículos científicos que aparecieron en la prensa española tras el 30 de abril. En él analizaba las consecuencias ecológicas de la catástrofe de Doñana. En El Mundo (4 de mayo) este investigador indicaba: "Nos guste o no, este episodio ha marcado inexorablemente un antes y un después. No es de extrañar que haya quienes lo minimicen, lo intenten marginar o no lo consideren. Se había avisado y denunciado desde hacía muchos años en muy diversas ocasiones y foros de todas las formas posibles, y guste o no, tarde o temprano aparecerán los responsables". En el artículo, Castroviejo criticaba alguna de las hipótesis propuestas para regenerar el entorno calificándolas como "aterradoras". 
El caso de Castroviejo es paradigmático de esta política típicamente española de prescindir de los verdaderos expertos. En el momento de suceder el accidente este investigador que tanto hizo por la fundación de la Estación Biológica de Doñana, era presidente del comité español del programa Hombre-Biosfera de la UNESCO -auspiciado por Naciones Unidas-, cuyo objetivo es potenciar y coordinar las investigaciones sobre medio ambiente que se llevan a cabo en todo el planeta.

$\mathrm{Su}$ calidad como investigador tenía tal prestigio en el ámbito científico internacional que en noviembre de 1998 fue elegido presidente del Consejo Internacional de Coordinación de los mencionados programas Hombre-Biosfera. Su función era coordinar los programas sobre investigación medioambiental que se llevan a cabo en todos los países del mundo.

En cualquier país occidental con tradición científica no cabe duda de que él hubiese sido el presidente del comité de expertos. En España ni siquiera se le invitó a que asistiera a la primera reunión. Además, desde el gabinete de prensa del CSIC se evitó que los periodistas pudieran ponerse en contacto con él remitiéndolos siempre a los expertos seleccionados por Nombela. Lo grave, desde el punto de vista del flujo de comunicación científica en España, no es que esto suceda, sino que la prensa libre española no tenga la especialización suficiente en ciencia y medio ambiente como para denunciar estas maniobras.

También llamó la atención que no hubiese investigadores de las universidades andaluzas. Desde el punto de vista institucional, causó perplejidad en medios científicos que ni siquiera estuviera, aunque sólo fuera como invitado sin derecho a opinar, el presidente de la Real Academia de Ciencias, Ángel Martín Municio ${ }^{11}$, quien además era un reputado bioquímico.

En el comité sí estaba, por ejemplo, Teresa Mendizábal, doctora en Físicas pero que llevaba muchos años de asesora de cuestiones protocolarias del CSIC, o Miguel García Guerrero, vicepresidente de Organización del CSIC. Fuentes de este organismo aseguraron que, en realidad, sólo 3 de los 17 "expertos" de Doñana habían dirigido trabajos en investigaciones relacionadas con el ecosistema del parque.

En concreto se trataban, además del director propuesto por Nombela, Miguel Ferrer, de los investigadores Fernando Hiraldo, vicedirector de la Estación Biológica, y de José Juan Chans, también de esta estación. No estaba, sin embar-

11 Ángel Martín Municio señaló en entrevista a quien suscribe este artículo que la "desastrosa" forma de seleccionar a los científicos integrantes en el comité de expertos en Doñana no tiene parangón en otros países desarrollados. Sin embargo, evitó pronunciarse más sobre este asunto "para que no me acusen de que yo quiero protagonismo". Añadió: "Lo de Doñana ha sido un asunto muy feo". 
go, Pablo Arambarri, profesor de investigación del CSIC quien ya en 1977 había alertado del riesgo del complejo minero de Aznalcóllar.

Quien suscribe este artículo no ha podido averiguar por qué el comité de expertos sólo lo constituían 17 de ellos y no 18 u otra cantidad. En una crisis como ésta, lo lógico es que se hubiese propiciado, incluso, una asamblea de científicos. Con el tiempo el número de científicos consultados fue aumentando -pero sólo en calidad de invitados-. Sin embargo, en los medios de comunicación, al menos en el periodo estudiado en Doñana -de abril a julio-, a partir de mediados de mayo sólo aparecen científicos externos al comité de expertos en una ocasión ( $E l$ Mundo, 6 de julio).

Algún periodista preguntó cómo era posible que Miguel Delibes o Javier Castroviejo no estuvieran desde el principio en el comité de expertos. La respuesta del gabinete fue que al estar tan implicados en la investigación de Doñana "sus conclusiones podrían no ser objetivas". Lo terrible, nuevamente, no fue la respuesta -en España parece que saben más de los temas aquellos que nunca los han estudiado- sino que el periodista la diera por válida. Así, a partir de la irrupción del CSIC en el escenario de la crisis, el resto de los científicos dejó de aparecer en los medios de comunicación. Para la prensa española sólo existieron sus miembros como referentes científicos.

En el periodo estudiado para la crisis de Doñana, desde el 25 de abril hasta el 31 de julio, César Nombela apareció en los medios en 81 ocasiones. Miguel Delibes, en cambio, sólo salió en cuatro, Martín Municio en tres y Castroviejo en una. El director en ese entonces de la Estación Biológica, Miguel Ferrer, apareció en 33 ocasiones. Destacan algunas declaraciones de César Nombela en apoyo claro al gobierno pero extendidas al CSIC que era como extenderlas a la "voz de la ciencia española". "Elogios del CSIC a las medidas de urgencia que decidió Medio Ambiente", (titular de $A b c, 3$ de mayo).

La estrategia había dado resultado. El criterio científico en vez de ser el consenso de universidades y academias españolas junto al CSIC estaba sólo manos de este último. Incluso se prohibió tomar muestras de los lodos para un posterior análisis químico a personas que no pertenecieran al CSIC. Así, cuando miembros de Greenpeace, intentaron recoger lodos para analizarlos, tanto desde la empresa como de la Junta o el gobierno se apresuraron a decir que esos análisis carecían de valor, aunque fuesen certificados por el Colegio Oficial de Químicos. El redactor especializado en medio ambiente de El Mundo, Gustavo Catalán, fue zarandeado y expulsado de la zona afectada cuando intentaba fotografiarla y obtener muestras del lodo, lo cual originó una denuncia de la Asociación Española de Periodistas de Información Ambiental (APIA).

Quien suscribe este artículo no ha podido averiguar cuáles fueron las verdaderas causas por las que las universidades andaluzas, en especial la de Sevilla, no tomaron una iniciativa científica al principio de la crisis y constituyeron un 
comité de expertos desde el primer momento ${ }^{12}$. Muchas opiniones coinciden en que tanto a la Junta como al ministerio le interesaban minimizar los efectos de la catástrofe y confiaban más en un organismo científico con presidencia política como el CSIC- que en uno independiente como las universidades. En este sentido, matizan que si la responsabilidad hubiese sido únicamente del Ministerio de Medio Ambiente, la Junta de Andalucía sí hubiese propiciado un comité de expertos en su comunidad autónoma desde el primer momento.

\section{Estrategia de comunicación para sacar partido de la crisis}

La estrategia del CSIC estaba clara. No se podía mentir, porque los resultados científicos son reproducibles y si se perdía la credibilidad científica del CSIC fallaba la base de la estrategia. Pero sí se podían dosificar los datos negativos, ocular algunos contaminantes y, sobre todo, dar la impresión a la opinión pública de que era un problema resoluble para la ciencia española. Todas sus comunicaciones tenían que tener un carácter "eminentemente positivo". Desde el CSIC no debían exigirse responsabilidades políticas, sino aportar soluciones. Sin embargo, también quedó muy claro desde el principio que en este pulso el CSIC se jugaba su prestigio científico de cara al futuro. Aparecer en primera escena de los medios de comunicación y en las portadas y editoriales de todos ellos representaba una oportunidad única para consolidar la imagen corporativa de prestigio. De hecho, el CSIC nunca había aparecido tanto en la prensa española como en mayo de 1998.

La fórmula para equilibrar el prestigio científico con la oportunidad de apoyar al gobierno que había nombrado a sus responsables, pasaba, ineludiblemente, por consultar a todos los verdaderos expertos ${ }^{13}$ posibles -tanto nacionales como internacionales- y elaborar un plan riguroso desde el punto de vista científico. Posteriormente, sobre ese plan se podría hacer las correcciones oportunas para los medios de comunicación, pero la idea central debía ser científicamente rigurosa. Para evitar que se desvelaran aspectos inoportunos de los estudios científicos se previó, precisamente, la necesidad de que sólo hicieran declaraciones los expertos seleccionados en un principio.

12 No obstante, el CSIC buscó la ayuda de las universidades andaluzas. Así, por ejemplo, los análisis de la contaminación de los suelos del Parque son realizados por el Departamento de Edafología de la Universidad de Granada. Su director, José Aguilar, uno de los catedráticos más antiguos y reconocidos en Edafología tampoco estuvo en el comité de expertos. La Universidad de Granada no da informes oficiales a la prensa. Todos los datos pasan por el Gabiente de Prensa del CSIC en Madrid.

13 Para ello se convocó un congreso en el que participaron algunos verdaders expertos como Miguel Delibes al que, no obstante, no invitaron al Comité del CSIC. 


\section{La entrada en escena}

En toda situación de crisis debe tenerse iniciativa e intentar neutralizar las opiniones no convenientes. Para ello se diseñó una estrategia en la que los "expertos" se reunían y emitían informes cada semana. En el gabinete de prensa se dio la orden tajante de que a los periodistas sólo se le podían facilitar los nombres que figuraban en el listado de expertos. Jamás otros investigadores, aunque tuvieran más experiencia científica en Doñana, como Delibes o Castroviejo. También se prohibió el nombre de Román Román, posiblemente como represalia por sus declaraciones en El Mundo (29 de abril) ya citadas.

La entrada en escena del CSIC fue muy bien acogida por los medios de comunicación, sobre todo, por los de ámbito nacional, ávidos de fuentes científicas que estuvieran en Madrid. Ninguno de ellos reparó en si los investigadores seleccionados eran los más adecuados ni se preguntó sobre los criterios utilizados para la elección de los miembros del comité. Tampoco se hizo alusión a sus currículos, a su idoneidad o falta de ella y, sobre todo, ninguno mencionó si faltaban o no investigadores españoles de prestigio. Ningún medio de comunicación puso en duda la autoridad del CSIC ni reclamó la presencia de científicos procedentes de las universidades españolas. El Mundo (30 abril) se refiere a la primera reunión celebrada por los expertos del Consejo en los siguientes términos "la denominada reunión Doñana, de carácter permanente, es en realidad un auténtico sanedrín de sabios". A continuación se habla de la talla profesional de los investigadores que la componen, para concluir definiendo a los integrantes de la comisión como "La flor y nata de la investigación oficial española". Desde ese momento, los expertos del CSIC se convierten para todos los periódicos españoles en el grupo o comité de "sabios". La entrada en juego del CSIC mereció grandes espacios en los medios de comunicación. El 29 de abril fue la reunión y el 30 es el día en que más informaciones aparecen sobre Doñana, con excepción de los primeros días de la catástrofe.

La difusión del segundo informe se hizo pública el 5 de mayo. Para lograr la atención de los medios, los expertos y miembros del gabinete de prensa se desplazaron hasta Doñana, pues desde allí estaban informando los redactores especializados en medio ambiente de la prensa española. La reunión tuvo lugar en el palacio de Doñana.

Analizando las declaraciones que aparecen en la prensa del día siguiente se demuestra cómo Nombela siguió al pie de la letra los consejos que desde el gabinete de prensa le fueron dados: "Sólo debe decir ideas positivas".

"Científicos y políticos: 'no hay motivo de alarma', fue el titular de $A b c$ del 6 de mayo. El Mundo destacaba en la entradilla de su información que ocupaba dos páginas completas unas declaraciones con tintes claramente políticos del presidente del CSIC: "Si la ciencia no actuó con celeridad cuando el síndrome 
tóxico, en esta oportunidad no va a ocurrir lo mismo", reprochando, de paso, la calidad de la ciencia que se hacía en épocas anteriores ${ }^{14}$.

En El Mundo (6 de mayo) también aparece por primera vez una de las frases positivas elaboradas por el gabinete de prensa y que a partir de ese momento repetiría con frecuencia César Nombela: "Reparar el daño ambiental causado por Doñana puede ayudar a restaurar la imagen de los científicos españoles". Y reiteraba: "Si la comunidad científica española falló cuando el síndrome tóxico esta vez no va a ser asî”.

Lo que era un desastre no sólo desde el punto de vista ecológico, sino en cuanto a dejación de responsabilidades de los políticos españoles se convertía, de repente, en la "oportunidad histórica" que tenían la ciencia española para reparar ante el mundo su mala imagen.

Nombela también desmintió en la rueda de prensa ofrecida tras la reunión del 5 de mayo las declaraciones del director de la Estación Biológica de Doñana, Miguel Ferrer, del día 29 de abril, en las que afirmaba que uno de los acuíferos que surten de agua Doñana estaba contaminado. No daba datos pero consideró "muy poco probable" la contaminación del mismo. En este caso se trataba de avalar las declaraciones de la ministra que había asegurado que el parque nacional no había sido dañado. Sin embargo, en su comparecencia del 27 de mayo en el Congreso de los Diputados junto con el director del Instituto Geominero de España -dependiente del Ministerio de Medio Ambiente-, Emilio Custodio, ambos reconocieron que el vertido de residuos tóxicos de la mina de Aznalcóllar afectó al acuífero 27 que abastece las marismas de Doñana.

Ambos matizaron estas afirmaciones señalando que la afección del acuífero 27 es muy pequeña y, por tanto, subsanable. (El Mundo, 28 de mayo).

14 En realidad, el objetivo era aprovechar la coyuntura para, de paso, desacreditar la ciencia que se hacía en la época socialista. Sin embargo, en rigor, la intoxicación por aceite de colza desnaturalizado se produjo en 1981. En decir, en la época de la UCD. En un principio el gobierno de Leopoldo Calvo Sotelo quiso, al igual que sucedió en 1998 con Doñana, quitarle importancia al asunto. El ministro de Sanidad de esa época, Jesús Sancho Rof, definió la enfermedad como "un bichito que si se cae de la mesa se mata". El "bichito" resultó ser una corruptela en la que existían muchos implicados -17 acusados- y cuyo objeto consistía en mezclar aceites de consumo humano con anilinas y aceites para otros usos, más baratos, pero que eran vendidos al consumidor al precio de aceite comestible. La primera muerte se produjo el 1 de mayo de 1981. En total murieron 234 personas y 16.000 resultaron intoxicadas. En un principio, como afectaba al sistema pulmonar, se la definió como neumonía atípica. En aquel episodio también fue el CSIC el encargado de realizar las investigaciones para esclarecer las causas de la enfermedad. El juicio se produjo en 1987, ya en la segunda legislatura socialista. Esta circunstancia y el hecho de que el suceso ocurrió a mediados de 1981, muy cerca de la primera legislatura socialista -1982- hicieron pensar al gabinete de prensa del CSIC que la mayoría de la opinión pública española lo atribuía, en realidad, al gobierno del PSOE. Por ello, no se consideraron contraproducentes, desde el punto de vista político, las afirmaciones de Nombela que, en realidad, desacreditaban al sistema científico de la España franquista y de la UCD, muchos de cuyos responsables estaban en el momento de producirse la catástrofe ecológica de Doñana en las filas del PP. 
Otro ejemplo de la postura del presidente del CSIC intentando suavizar la gravedad de la catástrofe está en sus declaraciones del jueves 30 de abril a la agencia Europa Press ${ }^{15}$, en la cual se mostraba "esperanzado" por el nivel "extremadamente bajo" de mercurio existente en los lodos tóxicos. Añadía: "Siendo éste uno de los metales más tóxicos, el resultado es bastante positivo, puesto que al ser más bajo de lo que mucha gente esperaba, aporta cierta esperanza para resolver el problema ${ }^{16 "}$.

\section{Cambios en la estrategia de comunicación}

Esa estrategia de consenso, de ideas eminentemente positivas y de informes favorables a las tareas de regeneración se fue modificando ligeramente a partir de finales de mayo cuando se observó que los trabajos de regeneración de los márgenes de la cuenca hidrográfica del Guadiamar, responsabilidad de la Confederación Hidrográfica del Guadalquivir, que a su vez pertenece al Ministerio de Medio Ambiente, iban mucho mejor que los de rehabilitación de las grandes superficies de suelos afectados pertenecientes a la Junta de Andalucía. Existen bastantes informaciones que demuestran esta hipótesis. Una de ellas es que mientras el CSIC alabó directamente los métodos empleados por el ministerio, nunca realizó comentarios similares respecto de los de la Junta de Andalucía. De hecho, criticó abiertamente la forma en que la Junta eliminaba los lodos -con palas y tractores- y recomendó que se hiciera a mano "para evitar que se pierdan las semillas de las capas de tierra superiores". Pero, obviamente, no era lo mismo retirar los lodos de la cuenca del río -10 metros desde cada orilla- a mano que hacerlo a lo largo de 5.000 hectáreas de extensiones agrícolas. Tanto César Nombela como Miguel Ferrer empezaron a denunciar que la retirada de los lodos "iba demasiado lenta" y que "la situación del parque se agrava día a día" ( $E l$ Periódico de Cataluña, La Vanguardia, El Mundo, El País, Abc, Diario 16, del 27 de junio).

\section{Los científicos andaluces intentan entrar en escena}

La iniciativa de actuación en la crisis estaba ahora en manos del CSIC y lo que en principio fueron propósitos de restar importancia a la catástrofe estaba tomando otro cariz. Esto hizo que la Junta de Andalucía, principal afectada ahora

15 Declaraciones publicadas, entre otros medios, por El Mundo, 1-5-1998: 26.

16 En estas declaraciones, Nombela evita pronunciarse sobre los niveles de otro metal pesado, el arsénico, cuyas concentraciones sí son más altas de las esperadas en un principio. Se espera una semana, 7 de mayo, para hacerlo público. Sin embargo, la intención no era difundirlo tan rápido, sino esperar a que la crisis amainara. Pero una filtración alertó a los periodistas de El Periódico de Cataluña, La Vanguardia y El Mundo. 
por las críticas del CSIC, exigiese que "Los informes del CSIC sobre el vertido tengan que pasar por el filtro de la Junta y del Gobierno" (Titular de El Mundo, 27 de junio). De hecho, estaba previsto difundir el informe sobre los niveles de contaminación atmosférica de metales pesados para el día 25 de junio. Sin embargo, fue "secuestrado" por el coordinador de las actuaciones del gobierno, Félix Pérez Meyer, quien en rueda de prensa explicó a los periodistas que, a partir de esa fecha, "los informes del CSIC sobre las consecuencias del vertido tóxico tendrán que ser 'traducidos' por el comité de coordinación de la Junta y del gobierno para no causar alarmas innecesarias en la población, no asustar y unificar criterios". (El Mundo, 27 de junio).

Desde el punto de vista de la teoría de la estrategia de la manipulación informativa en tiempos de crisis, la Junta de Andalucía estaba pagando caro el hecho de no haber planteado desde el principio un comité de expertos andaluces afín a sus intereses. Para remediar esta situación, nombró en junio al catedrático de Ecología de la Universidad de Sevilla Francisco García Novo, coordinador del grupo de catedráticos y profesores andaluces "excluidos del comité de sabios" ( $E l$ Mundo, 6 de julio). Como en toda crisis informativa, una vez transcurrido los primeros momentos, los que tomaron la iniciativa en un principio se consolidan como protagonistas de la información y el resto deja de ser relevante. En este caso también se demuestra que, al menos en los diarios de ámbito nacional, este grupo de expertos andaluces apenas salió en ellos.

A juzgar por las declaraciones de García Novo, da la impresión de que el objetivo de esta comisión era plegarse a los intereses de la Junta, es decir justificar intereses políticos, y no simplemente un aporte de ideas a la comunidad científica. Un ejemplo: en una información publicada por El Mundo (6 de julio) se señala que en el último informe del CSIC se indica: "El río Guadiamar se recupera más lentamente de lo esperado". Sin embargo, para García Novo, citado en esa misma información, "el río se regenera de manera satisfactoria en todos sus tramos", de acuerdo con su propio informe. Mientras García Novo señalaba que a finales de mayo ya había colonización de algas e insectos, para el CSIC esto sucedía porque "estos insectos ponen huevos en el río y, como no hay otros animales, su supervivencia es alta". Sin embargo, se reafirmaban en que no había recolonización del río y que la prueba era, precisamente, la presencia de algas e insectos. Estas contradicciones entre los científicos servían a los políticos para desacreditar actuaciones que los propios investigadores proponían. Era una estrategia de comunicación diseñada por el gabinete de la Junta que se basaba en crear en la opinión pública española la imagen de que existía desacuerdo entre los científicos, de forma que los políticos pudieran actuar sin tener en cuenta sus recomendaciones $\mathrm{y}$, encima, no ser criticados por ello. 
Periodismo especializado en medio ambiente:

el caso Doñana como paradigma de manipulación informativa

\section{Lucha entre intereses científicos y políticos: el desembalse de agua de Entre- muros}

En muchos episodios en los que los políticos quieren manipular a los científicos, llega un momento en el que los investigadores se rebelan. Esto suele suceder cuando se les intenta forzar para que revistan de carácter científico una decisión política que no se sostiene desde el punto de vista de la teoría científica aceptada.

En estos casos los investigadores deben elegir entre el prestigio científico y el poder político y casi siempre suelen elegir el primero, entre otros motivos, porque el prestigio científico es muy difícil de conseguir y muy fácil de perder. Sin embargo, en un sistema democrático el poder político no es excesivamente difícil de conseguir, sobre todo, si se tiene prestigio social y profesional.

Uno de los ejemplos más ilustrativos de esta lucha entre intereses políticos y científicos sucedió con el informe del CSIC del 5 de junio en el cual se recomendaba la liberación al Guadalquivir de las aguas ácidas retenidas en el embalse de Entremuros, una vez que se les hubiese rebajado su grado de acidez. El plan del CSIC preveía que los 4,5 hectómetros cúbicos ${ }^{17}$ de agua ácida debían ser trasladadas, poco a poco, a una balsa de depuración, situada junto al último dique de contención, donde recibiría un tratamiento con hidróxido de calcio. El agua se distribuiría a modo de lechada en el Guadalquivir y se necesitaban 1.600 toneladas de hidróxido de calcio para aumentar el $\mathrm{pH}$-es decir, disminuir la acidez- del agua embalsada. Todo este proceso debía hacerse antes de que llegaran las lluvias de otoño y la balsa reventara.

A la ministra Isabel Tocino no le gustaba la idea de verter algo en el Guadalquivir pues ella -su ministerio- era la responsable de preservar el buen estado de los ríos españoles. Sabía que si sucedía algo malo, la Junta de Andalucía comenzaría una gran estrategia mediática contra la ministra e, incluso, contra los científicos del CSIC a modo de venganza.

La ministra conocía de antemano el informe del CSIC antes de que éste se divulgara a la prensa. No supo qué hacer, desde el punto de vista de la estrategia de comunicación, y su instinto le hizo desmarcarse de los expertos del CSIC que, en otras ocasiones, tanto la habían apoyado. "Tocino cuestiona el último informe de los expertos del CSIC sobre la retirada de los lodos" (Titular de portada de $E l$ Mundo en Andalucía, 7 de junio). El texto de la información citada señalaba:

17 Resulta interesante, desde el punto de vista de técnicas de manipulación del lenguaje, el hecho de que tanto desde el Ministerio de Medio Ambiente, la Junta de Andalucía y el CSIC siempre se hiciera referencia a 4,5 hectómetros cúbicos, una medida, la del hectómetro cúbico, poco usada y que, en la mayoría de la población, da lugar a una representación abstracta de la verdadera cantidad de agua tóxica. En realidad 4,5 hectómetros cúbicos son 4.500 millones de litros de agua. Pero, obviamente, esa cantidad es más impactante. En las informaciones publicadas por la prensa española analizadas siempre aparece la cantidad en hectómetros cúbicos, aunque, a veces se habla de "millones de litros" embalsados, pero nunca se especifica cuántos son. 
"Aparentemente, en el desembalse del agua tóxica de Entremuros la ministra no ha confiado en los estudios que durante un mes y medio han hecho los expertos del CSIC para llevar a cabo la depuración de los 4,5 hectómetros allí embalsados, ya que se ha encargado otro a la empresa Imerco. La ministra llegó a pedir al presidente del CSIC, César Nombela, 'que explique qué quieren decir con éste último informe'. Isabel Tocino manifestó que desde el primer momento la Confederación Hidrográfica del Guadalquivir ha trabajado de acuerdo con las pautas que le han marcado los expertos. Pero en el tema del desembalse, después de llegar a un acuerdo sobre la necesidad de depurar esas aguas -que niega el director del parque nacionalel informe del CSIC sobre Entremuros ni se menciona en las reuniones". ( $E l$ Mundo, edición de Andalucía, 7-6-1998, portada)

El asunto del desembalse de Entremuros provocó una de las situaciones más tensas en el CSIC. Muchos piensan que si el nombramiento del presidente del CSIC hubiese dependido de la ministra de Medio Ambiente -en el momento en el que sucedieron estos acontecimientos el nombramiento dependía de la ministra de Educación y Cultura-, Isabel Tocino lo hubiese cesado o lo hubiese obligado a plegarse más a sus intereses.

Pero, en este caso, creo que el espíritu de científico de César Nombela pudo más que su apego al cargo. Normalmente los investigadores que llevan una vida dedicados al estudio riguroso de cualquier aspecto de la ciencia o la cultura tienen un límite respecto a la manipulación de sus ideas. Esta opinión, generalizada en algunos ámbitos políticos, es la que justifica, por ejemplo, que la ministra de Medio Ambiente haya preferido elegir para el cargo de director del Instituto Nacional de Meteorología a Eduardo Coca, un abogado técnico del estado, en lugar de a un científico ${ }^{18}$.

Sin embargo, desde el punto de vista institucional del CSIC, su enfrentamiento con la ministra le otorgó una apariencia de independencia muy valorada por los medios de comunicación y que ha ayudado a consolidar su imagen corporativa. En cuanto al conocimiento del CSIC por la opinión pública española

18 En el diario El País (25 enero de 2000) aparece una denuncia de una comisión de meteorólogos del INM porque Eduardo Coca se arrogue el derecho de opinar como científico ante los medios de comunicación. En concreto, se le critica sus opiniones -calificadas como «sin fundamento"- sobre los aerolitos caídos en la península ibérica a mediados de enero. Una vez más, desde esta tesis doctoral se indica que el problema no reside tanto en el nombramiento, cuyo objetivo muchas veces es el de plegarse a los intereses políticos, sino en la falta de periodistas especializados que no indagan sobre el currículo de las fuentes y presentan en este caso a Eduardo Coca como autoridad científica. En este sentido, es relevante cómo Eduardo Coca era en 1998 la fuente habitual de la agencia Efe para explicar científicamente los fenómenos atmosféricos que de forma habitual desencadenan temporales en España. Para Efe, es más relevante el cargo que la autoridad científica, en un asunto eminentemente científico. 
es evidente que existe un antes y un después desde la catástrofe ecológica de Doñana.

Lo que sigue es un breve resumen de la aparición destacada del CSIC en los medios de comunicación durante el periodo estudiado en esta tesis: El País (29 de abril) publica una entrevista destacada en portada con Miguel Ferrer. El Periódico de Cataluña (30 de abril) dedica a este asunto un editorial titulado "Las cosas como son". El mismo diario (8 de mayo) vuelve a publicar otro editorial: "Isabel Tocino no se explica sobre Doñana", en el que elogia la actuación del CSIC. El 10 de mayo aparece en $A b c$, en la sección tribuna abierta, un artículo de César Nombela titulado "Aznalcóllar: serenidad y rigor". Ese mismo día la revista de $E l$ Mundo dedica un reportaje de 10 páginas al accidente ecológico. La Vanguardia (10 de mayo) publica una entrevista, destacada en portada, con Miguel Ferrer. El 11 de mayo las revistas Epoca (6 páginas), Cambio 16 (8 páginas) y Tribuna (7 páginas), publican reportajes sobre Doñana en los que mencionan al CSIC. El Magazine de La Vanguardia dedica un reportaje de 7 páginas: "Doñana: el paraíso asediado". El Periódico de Cataluña (17 de mayo) publica un editorial titulado "Doñana o la pérdida de credibilidad", elogiando al CSIC. La Vanguardia (17 de mayo) entrevista a César Nombela. El Semanal de El País (24 de mayo) dedica un reportaje de 9 páginas a "Doñana, el paraíso amenazado". El País (2 de julio) y El Mundo (11 de julio) publican sendas entrevistas con César Nombela.

\section{Los informes: mantener a los periodistas entretenidos}

Los informes ${ }^{19}$ elaborados por la comisión de expertos del CSIC se convirtieron desde el primer momento en la información más demandada por los medios. En la teoría sobre estrategias de resolución de crisis informativas se indica que en ellas "se tiene horror al vacío". En la gigantesca aspiración de datos por el saber, los medios de información, entregados a sí mimos, se benefician a priori de la iniciativa, y siempre la agradecen.

Los informes servían en unas ocasiones como argumento principal sobre el que se apoyan todas las noticias, y en otras, como referencia para evaluar las actuaciones que se desarrollaban en la zona. La identificación de los medios de comunicación con los informes llegó hasta tal punto que los procesos de retirada de lodos que no se habían realizado siguiendo los consejos del CSIC son denunciados por los medios de comunicación (La Vanguardia, 13 de mayo y El País, 9 de junio).

19 Hasta el momento de redactar la tesis de la que se obtiene este artículo, el CSIC había publicado 13 informes. La secuencia es la siguiente: 29 de abril, 5 de mayo, 12 de mayo, 22 de mayo, 5 de junio, 25 de junio, 23 de septiembre, 2 de octubre y 26 de noviembre de 1998. En 1999 se publicó uno el 9 de marzo, otro el 3 de mayo y el último de ese año el 23 de diciembre. Al finalizar 1998 se publicaron las conclusiones de un seminario sobre Doñana, que también pueden considerarse como un informe de la situación. 
Cuando la opinión de los expertos del CSIC es acogida por las distintas administraciones con alguna reserva, los medios destacan que "se quiere acabar con las voces que molestan" (El Mundo, 26 de mayo, editorial). En más de una ocasión los medios apuestan por que se cumplan las recomendaciones del CSIC (El Mundo, 7 de junio). Cuando aparecen opiniones contradictorias, los periódicos no se pronuncian "hasta que el Consejo Superior de Investigaciones Científicas emita su dictamen" (El País, 8 de mayo y Abc, 9 de mayo). El diario El País (2 de junio) destacaba "la buena acogida que están teniendo los informes periódicos de los expertos". La incidencia de los informes en los medios es patente, sobre todo la del tercer informe reflejado en la prensa el 13 y 14 de mayo y la del quinto el 7 de junio.

Desde el punto de vista de la comunicación, la importancia de los informes radicaba en que servían como alimento informativo para los periodistas, eclipsando otras posibles fuentes. Desde el 29 de abril hasta el 25 de junio, cuando la crisis estaba en su apogeo, se publicó un informe cada 10 días. En mayo -el mes en que informativamente este asunto fue más relevante- se difundieron 4 informes ${ }^{20}$ del comité de expertos. En junio se publicaron dos. En verano decayó el interés y no se volvió a publicar el otro hasta el 23 de septiembre.

Podría argumentarse que los informes se elaboraban no en función de las necesidades de alimentación de los medios de comunicación, sino de la pura obtención de datos científicos. Pero esto no se sostiene, puesto que lo habitual en el método científico es que los resultados se obtengan al final, de forma que lo normal hubiese sido que la producción de informes hubiese sido superior en los meses de julio y agosto cuando se hubiesen obtenido y analizado todos los datos. No se justifica que al comienzo de una investigación científica seria se elaboren tantos informes y, al final, decrezca su número.

\section{La recompensa de la estrategia: rigor y credibilidad para el CSIC}

La recompensa de la estrategia del gabinete de comunicación fue la obtención de unos niveles de rigor y credibilidad para el CSIC desconocidos hasta ese momento. La credibilidad en los medios resulta patente. El Periódico de Cataluña (17 de mayo) alude al Consejo en un editorial, en los siguientes términos:

"Los españoles necesitamos que dejen de mentirnos sobre Doñana. Urge designar una voz única y no manipulada que informe sobre los daños. Pero, ¿de dónde sacamos una voz libre que no se sienta condicionada por las responsabilidades que les corresponden en la catástrofe a los poderes políticos? Está claro que el culpable directo de la rotura fue la empresa Boliden,

20 Se incluye aquí también el del 29 de abril. 
explotadora de las minas. Pero la mala función autorizadora y supervisora de las administraciones clama al cielo, y la torpeza en la respuesta tras el desastre merece destituciones. Afortunadamente, hay gente que trabaja con seriedad y que, sin intención alarmista, advierte de los peligros presentes y futuros. Ni la Universidad de Barcelona, que ha analizado los lodos, ni el Consejo Superior de Investigaciones Científicas son cosa de aficionados. Ambas instituciones certifican la alta contaminación de los fangos y advierten que podría haber daños irreparables. El CSIC denuncia incluso el peligro que puede suponer para la salud la inhalación de las partículas metálicas contenidas en el barro, e insta a los políticos a que se retiren los lodos a un ritmo tres veces superior al actual. Cuando ya han pasado tres semanas desde el accidente, y ante la falta de credibilidad de las versiones oficiales, la opinión pública española continúa creyendo más en las versiones independientes que publican los medios de comunicación que en las oficiales" (El Periódico de Cataluña, 17-5-1998: 3)

En la misma línea se muestra el diario El País (8 de mayo), donde señalaba: "Hay que huir de alarmismos", remitiéndose después al informe que estaba realizando el CSIC. Los medios destacan los criterios del CSIC que fueron establecidos desde el principio y que con el paso del tiempo se ha demostrado su rigor y solvencia. Así por ejemplo, cuando desde la Junta de Andalucía se tildó la propuesta del CSIC -de desaconsejar el paso por el Vado del Quema- como "alarmista" los medios resaltan (La Vanguardia, El País, El Mundo, $A b c, 15$ de mayo) que la Junta obliga a cambiar uno de los trayectos de la romería del Rocío como propuso en su día el CSIC. Titulares como "El Gobierno recurre para paliar la catástrofe de Doñana a expertos del CSIC cuyas alertas ignoró" ( $A b c, 1$ de junio) o "Los científicos ganan el duelo del desembalse de las aguas tóxicas" (El Mundo, 26 de mayo), ponen de manifiesto la credibilidad que los medios dieron desde el primer momento a las opiniones del CSIC. Cuando surge un nuevo problema relacionado con el accidente de Aznalcóllar, los medios ya lo ven como «otro reto que tendrá que afrontar la Comisión de Expertos" (El País, 10 de mayo). Incluso, cuando se tarda en aceptar sus recomendaciones se dice:

"Pese a que el tratamiento de esta aguas antes de bombardearlas hacia el Guadalquivir fue una de las primeras recomendaciones de los expertos del CSIC, hasta ahora nada se había hecho al respecto". (El Mundo, 26-5-1998: 30)

La estrategia había funcionado, pues el CSIC de casi no aparecer en los medios de comunicación al comienzo de la crisis, se había convertido en el único 
referente aceptado no sólo por éstos, sino por otros agentes sociales incluidos los ecologistas, tras decidir entrar en el escenario comunicacional.

La información sobre el accidente ecológico de Aznalcóllar acaparó la atención de los medios de comunicación, hasta el punto que de en mayo doblaron su espacio medio dedicado a la información ambiental, pasando del 2 por ciento que ocupa normalmente esta información en los grandes diarios nacionales al 4, 2 por ciento ${ }^{21}$.

De las 454 informaciones ${ }^{22}$ analizadas en nuestro periodo de estudio (abriljulio), 246 (53 por ciento) citan en su contenido al CSIC. Sin embargo, en el periodo de máxima atención informativa -del 26 de abril al 30 de junio- se publicaron 407 informaciones de las que 224 (55 por ciento) citan al CSIC; en este último periodo César Nombela aparece en 74 ocasiones.

\section{La criticable dependencia de los medios españoles hacia los gabinetes de prensa}

El profesor de la Universidad del País Vasco Txema Ramírez ha realizado varias investigaciones, cuyos resultados cita en su libro Gabinetes de Comunicación ${ }^{23}$, que indican que la dependencia de los medios españoles hacia los gabinetes de comunicación había crecido un 22 por ciento en 14 años. Así, en 1978, el 30 por ciento de las noticias seleccionadas y publicadas en los diarios estudiados El Correo Español, Deia, Egin, El País y Abc-tenían como fuente a un gabinete de prensa; en 1992, este porcentaje se había incrementado al 52 por ciento.

En otro de sus estudios sobre la influencia de los gabinetes de prensa en los medios de comunicación -realizado en 1992 sobre 60 periodistas de diversos medios españoles, responsables de seleccionar de entre la información que reciben, aquella susceptible de difundirse (gatekeepers)- se puso de manifiesto, entre otros datos, que un 32,23 por ciento de los encuestados consideraba que los gabinetes de prensa tienen mucha influencia en los medios de comunicación y un 39,04 opinaba que la influencia era "bastante". Según los encuestados, los comunicados que más se difundían eran los procedentes de gabinetes de comunicación del mundo económico y de partidos políticos y sindicatos (un 70,6\%), seguidos de los de gabinetes institucionales $(67,2 \%)$.

21 Datos obtenidos del análisis mensual que realiza el centro de estudios de Información Ambiental de Cataluña.

22 Para este análisis se han incluido los periódicos estudiados en la tesis de quien suscribe este artículo. Sin embargo, también se ha añadido la información proveniente de revistas de información general -Tiempo, Época, Cambio 16, Tribuna- y de los diarios económicos Cinco Días, Expansión y La Gaceta de los Negocios.

23 Ramírez, Txema. Gabinetes de comunicación. Bosch-Comunicación, Barcelona, 1995 
Sin embargo, en cuanto a la eficiencia, definida ésta como la capacidad que tiene cada gabinete de conseguir sus objetivos previstos, la investigación de Txema Ramírez determinó que eran los gabinetes institucionales, con un 85, 9 por ciento, los que tenían un mayor grado de esta cualidad, seguidos por los de los partidos políticos y sindicatos $(84,2 \%)$, el mundo económico $(80,7 \%)$ y, a bastante distancia, los gabinetes de comunicación de los movimientos sociales y ONGs, con un 35,1 por ciento de eficiencia y los de asesorías de comunicación, con un 31,5 por ciento.

La mayor parte de los gatekeeper encuestados valoraba como justo el tratamiento dado por los medios a las informaciones procedentes de los gabinetes, aunque un 38 por ciento lo consideraba "excesivo" o "desproporcionado".

Para algunos, estas actuaciones de los gabinetes de prensa no son éticamente reprochables, Pero sí lo es, desde todos los puntos de vista, el hecho de que los medios de comunicación se fíen tanto de los gabinetes institucionales españoles.

$\mathrm{Al}$ igual que los periodistas lucharon en tiempos pasados por la libertad de expresión y de información, su objetivo debería ser ahora enfrentarse a estos gabinetes. Si no lo hacen dará la impresión de que, en realidad, no se luchó por esa libertad de prensa para beneficiar a la sociedad sino, simplemente, porque esta libertad facilitaba su trabajo. Los gabinetes de prensa representan en la actualidad uno de los mayores peligros contra la libertad de información, pero como facilitan el duro trabajo diario del periodista, éstos no sólo no los critican sino que, además, los utilizan y los elogian.

En realidad, y tras analizar el trabajo que se llevaba a cabo en el gabinete de prensa del CSIC, puede afirmarse que los periodistas que trabajan en la actualidad en algunos de estos gabinetes podrían considerarse como los herederos de los antiguos censores de la época franquista. La diferencia estriba en que mientras los censores eran criticados y hasta vilipendiados por los sectores progresistas de la sociedad española, desde los profesores universitarios hasta los redactores y directores de periódicos, los modernos periodistas que trabajan en gabinetes de prensa son admirados por sus colegas y no sólo son aceptados por la sociedad, sino hasta encumbrados por ella. En muchos casos, estar al frente de un gabinete de prensa está considerado en la España de finales del siglo XX como un magnífico colofón a una carrera profesional como periodista.

Otra diferencia estaría en que mientras los antiguos censores eran reclutados en atención a sus méritos morales, los actuales responsables de gabinetes de prensa lo son en función de sus méritos profesionales y de la capacidad que puedan tener para poner los intereses de su patrón por delante de los principios éticos, como quedó aquí demostrado cuando a personas ajenas al trabajo de científicos se les puso una bata blanca para aparentar movilidad en un laboratorio cuyos investigadores no estaban dispuestos a dejarse manipular ante las cámaras de televisión. De 
forma que, como se ha podido demostrar en este capítulo, en algunos aspectos, su eficacia para ocultar información es mucho mayor que la de los censores franquistas.

(Recibido el 5-6-2000, aceptado el 15-6-2000) 